\title{
Quantitative Assessment of Elagolix Enzyme-Transporter Interplay and Drug-Drug Interactions Using Physiologically Based Pharmacokinetic Modeling
}

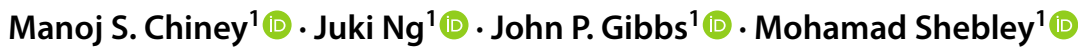

Published online: 12 November 2019

(c) The Author(s) 2019

\begin{abstract}
Introduction Elagolix is approved for the management of moderate-to-severe pain associated with endometriosis. The aim of this analysis was to develop a physiologically based pharmacokinetic (PBPK) model that describes the enzyme-transporter interplay involved in the disposition of elagolix and to predict the magnitude of drug-drug interaction (DDI) potential of elagolix as an inhibitor of P-glycoprotein (P-gp) and inducer of cytochrome P450 (CYP) 3A4.

Methods A PBPK model (SimCYP ${ }^{\circledR}$ version 15.0.86.0) was developed using elagolix data from in vitro, clinical PK and DDI studies. Data from DDI studies were used to quantify contributions of the uptake transporter organic anion transporting polypeptide (OATP) 1B1 and CYP3A4 in the disposition of elagolix, and to quantitatively assess the perpetrator potential of elagolix as a CYP3A4 inducer and P-gp inhibitor.

Results After accounting for the interplay between elagolix metabolism by CYP3A4 and uptake by OATP1B1, the modelpredicted PK parameters of elagolix along with the DDI $\mathrm{AUC}_{\infty}$ and $C_{\max }$ ratios, were within 1.5-fold of the observed data. Based on model simulations, elagolix $200 \mathrm{mg}$ administered twice daily is a moderate inducer of CYP3A4 (approximately $56 \%$ reduction in midazolam $\mathrm{AUC}_{\infty}$ ). Simulations of elagolix $150 \mathrm{mg}$ administered once daily with digoxin predicted an increase in digoxin $C_{\max }$ and $\mathrm{AUC}_{\infty}$ by $68 \%$ and $19 \%$, respectively.

Conclusions A PBPK model of elagolix was developed, verified, and applied to characterize the disposition interplay between CYP3A4 and OATP1B1, and to predict the DDI potential of elagolix as a perpetrator under dosing conditions that were not tested clinically. PBPK model-based predictions were used to support labeling language for DDI recommendations of elagolix.
\end{abstract}

\section{Introduction}

Elagolix is a non-peptide, oral gonadotropin-releasing hormone $(\mathrm{GnRH})$ receptor antagonist recently approved for the management of moderate to severe pain associated with endometriosis. Elagolix inhibition of GnRH receptors at the anterior pituitary level causes a rapid decrease in secretion of the gonadotropin luteinizing hormone (LH) and follicle-stimulating hormone (FSH), resulting

Electronic supplementary material The online version of this article (https://doi.org/10.1007/s40262-019-00833-6) contains supplementary material, which is available to authorized users.

Mohamad Shebley

mohamad.shebley@abbvie.com

1 Clinical Pharmacology and Pharmacometrics, AbbVie, Department R4PK, Building AP31-3, 1 North Waukegan Road, North Chicago, IL 60064, USA in a dose-dependent decrease in the ovarian production of estradiol and other sex hormones, with partial suppression at lower doses of elagolix, to nearly full suppression with higher doses [1]. The phase III clinical studies of elagolix demonstrated that elagolix $150 \mathrm{mg}$ once daily (QD) and $200 \mathrm{mg}$ twice daily (BID) produce clinically meaningful pain reduction, including dysmenorrhea and non-menstrual pelvic pain in premenopausal women with moderate to severe endometriosis-associated pain [2].

Elagolix is rapidly absorbed upon oral administration, with maximum plasma concentration $\left(C_{\max }\right)$ occurring $1 \mathrm{~h}$ after dosing. Elagolix exposures $\left[C_{\max }\right.$ and area under the concentration-time curve (AUC)] increase dose proportionally at the clinical doses of $150 \mathrm{mg}$ QD and $200 \mathrm{mg}$ BID, with an apparent terminal elimination half-life $\left(t_{1 / 2}\right)$ of approximately 4-6 h [3]. The contribution of the drugmetabolizing enzyme cytochrome P450 (CYP) 3A, efflux transporter P-glycoprotein (P-gp), and uptake transporter organic anion transporting polypeptide (OATP) 1B1/1B3 


\section{Key Points}

A whole-body physiologically based pharmacokinetic (PBPK) model of elagolix was developed to quantitatively account for the contribution of metabolizing enzymes, transporters, and their interplay.

The PBPK model was able to adequately describe clinical pharmacokinetics data and recover the observed drug-drug interaction (DDI), demonstrating that the model is verified for the intended purpose.

Simulations using elagolix PBPK model informed labeling information regarding the DDI potential with digoxin (P-glycoprotein substrate) and midazolam (cytochrome P450 3A4 substrate) under dosing conditions that were not tested clinically.

to the disposition of elagolix has been quantified based on mechanistic drug-drug interaction (DDI) studies of elagolix with ketoconazole (a strong inhibitor of CYP3A/P-gp) and rifampin (a CYP3A and P-gp inducer and OATP1B1/1B3 inhibitor) [4]. Following the coadministration of ketoconazole $400 \mathrm{mg}$ with elagolix $150 \mathrm{mg}$, the elagolix AUC increased 2.2-fold, indicating that elagolix is not a sensitive substrate of CYP3A4. Following a single dose of rifampin $600 \mathrm{mg}$ coadministered with elagolix $150 \mathrm{mg}$, the elagolix AUC increased 4.6-fold, while multiple doses of rifampin increased the elagolix AUC by 1.65-fold. The increase in elagolix exposures after single dosing may be attributable to OATP1B1 inhibition, while the decreased magnitude of the interaction of elagolix with multiple doses of rifampin are likely due to the net effect resulting from acute OATP1B1 inhibition and CYP3A/P-gp induction. Given that hepatic elimination is an important mechanism in the overall clearance of elagolix, hepatic impairment (HI) was associated with increased elagolix exposures in Child-Pugh B (AUC ratio 2.7) and Child-Pugh C (AUC ratio 6.7) patients compared with healthy volunteers. There was no impact on elagolix exposures in Child-Pugh A patients [5].

As a perpetrator, elagolix $150 \mathrm{mg}$ QD induced CYP3A based on the observed 35\% decrease in midazolam AUC, and at $200 \mathrm{mg}$ BID inhibited P-gp based on the observed approximately $70 \%$ and $30 \%$ increases in digoxin $C_{\max }$ and AUC, respectively. However, the effect of elagolix as a CYP3A inducer at a $200 \mathrm{mg}$ BID dose and as a P-gp inhibitor at a $150 \mathrm{mg}$ QD dose was not evaluated in clinical DDI studies $[6,7]$.

Physiologically based pharmacokinetic (PBPK) modeling is a powerful approach being used to predict the magnitude of DDI potential and is accepted by drug regulatory agencies
$[8,9]$. The application of PBPK to inform dosing recommendations and assessment of DDI in regulatory submissions is a fundamental part of the model-informed drug development paradigm. PBPK models that have been verified with clinical data have been increasingly used to predict the DDI potential for untested scenarios $[10,11]$. Given the regulatory acceptance and demonstrated scientific robustness of the PBPK approach, a PBPK model of elagolix was developed and verified to inform the DDI potential of elagolix in support of labeling recommendations.

Thus, the aim of the current analysis was to (1) develop and verify a whole-body PBPK model of elagolix that described the interplay between metabolism by CYP3A4, and hepatic uptake by OATP1B1, using clinical data from phase I PK single and multiple ascending doses, DDIs, and special populations studies of elagolix; and (2) predict the DDI potential of elagolix as a P-gp and CYP3A modulator at clinically relevant doses that were not evaluated in clinical DDI studies.

\section{Materials and Methods}

The SimCYP ${ }^{\circledR}$ (V15.0.86.0; Certara, Sheffield, UK) population-based absorption, distribution, metabolism, and excretion (ADME) simulator was used for the PBPK model development, verification, and application. The PBPK model of elagolix was developed using a combined bottom-up and top-down modeling approach [12]. The base model of elagolix was developed using the in vitro parameters, while the PK and DDI data from multiple clinical studies were used to optimize the contribution of each pathway involved in the ADME of elagolix. The final model parameters and their sources are summarized in Table 1.

\subsection{Elagolix Physiologically Based Pharmacokinetic (PBPK) Model Development, Verification, and Application}

SimCYP ${ }^{\circledR}$ library model files of ketoconazole, rifampin, midazolam, and digoxin were used during the elagolix PBPK model development to assign contributions of various disposition mechanisms. No modifications were made to these model files, except for rifampin, as described in Sect. 2.2, to capture the induction of P-gp following multiple dosing of rifampin. Figure 1 provides an overview of the strategy used for the elagolix PBPK model development, verification, and application. The base PBPK model of elagolix was initially developed using physicochemical and in vitro data (Table 1). The absorption of elagolix was captured using the mechanistic advanced dissolution, absorption, and metabolism (ADAM) model in SimCYP ${ }^{\circledR}$ [23]. The ADAM model was selected to capture the intestinal absorption, metabolism, 
and efflux of elagolix via CYP3A4 and P-gp. Parameters for the ADAM model were based on built-in predictive in silico tools within the simulator, in vitro permeability Caco-2 data, or were optimized based on clinical PK data (Table 1). Since elagolix is a substrate of the hepatic uptake transporter OATP1B1, a whole-body PBPK model functionality was used to capture the hepatic uptake transport kinetics in SimCYP ${ }^{\circledR}$ to describe the PK profile of elagolix in healthy volunteers. The apparent volume of distribution at steady state $\left(V_{\mathrm{ss}}\right)$ was estimated using the method published by Rodgers and Rowland (Method 2 in SimCYP ${ }^{\circledR}$ ) [13]. Since elagolix is actively transported into the hepatocyte via the uptake transporter OATP1B1, distribution to the liver was described by a permeability-limited model. The contribution of OATP1B1 to the overall uptake was estimated using in vitro and clinical data from a DDI study
Table 1 Input parameters of the elagolix PBPK model using SimCYP (V15.0.86.0)

\begin{tabular}{|c|c|c|}
\hline Parameter & $\begin{array}{l}\text { Parameter value used in } \\
\text { the SimCYP model }\end{array}$ & Source \\
\hline \multicolumn{3}{|l|}{ Physiochemical and blood binding } \\
\hline Molecular weight ( $\mathrm{g} / \mathrm{mol})$ & 631.6 & Experimental data \\
\hline $\log \mathrm{P}$ & 1.34 & \\
\hline $\mathrm{pK}_{\mathrm{a}} 1$ & 4 & \\
\hline $\mathrm{pK}_{\mathrm{a}} 2$ & 7.9 & \\
\hline Blood-to-plasma ratio & 0.62 & \\
\hline$f_{\mathrm{u}}$ & 0.21 & \\
\hline Absorption model & ADAM & \\
\hline Peff,man type & Regional & \\
\hline P Caco-2 $\left(10^{-6} \mathrm{~cm} / \mathrm{s}\right)$ & 10 & Estimated based on clinical data \\
\hline Input form & Solution & Experimental data \\
\hline Distribution model & Full PBPK model & \\
\hline$V_{\text {ss }}$ input type & Predicted using Method 2 & SimCYP predicted \\
\hline \multicolumn{3}{|l|}{ Elimination } \\
\hline CYP3A4 CL $\mathrm{Cint}_{\mathrm{i}}(\mu \mathrm{L} / \mathrm{min} / \mathrm{pmol}$ of isoform $)$ & 0.3 & Estimated based on clinical data \\
\hline CYP2C8 $\mathrm{CL}_{\mathrm{int}}(\mu \mathrm{L} / \mathrm{min} / \mathrm{pmol}$ of isoform $)$ & 0.029 & Experimental data \\
\hline CYP2D6 $\mathrm{CL}_{\text {int }}(\mu \mathrm{L} / \mathrm{min} / \mathrm{pmol}$ of isoform $)$ & 0.7 & Experimental data \\
\hline $\mathrm{CL}_{\text {renal }}(\mathrm{L} / \mathrm{h})$ & 1.6 & Estimated based on clinical data \\
\hline Additional systemic CL/F (L/h) & 9 & Estimated based on clinical data \\
\hline \multicolumn{3}{|l|}{ Transporter kinetics } \\
\hline \multicolumn{3}{|l|}{ P-gp } \\
\hline $\mathrm{CL}_{\text {int }}(\mu \mathrm{L} / \mathrm{min} / \mathrm{pmol})$ & 4 & Estimated based on clinical data \\
\hline \multicolumn{3}{|l|}{ OATP1B1 } \\
\hline$J_{\max }(\mathrm{pmol} / \mathrm{min} /$ million cells $)$ & 215 & Estimated based on clinical data \\
\hline$K_{\mathrm{m}}(\mu \mathrm{M})$ & 0.66 & Experimental data \\
\hline \multicolumn{3}{|l|}{ Perpetrator properties } \\
\hline \multicolumn{3}{|l|}{ CYP3A4 enzyme MBI } \\
\hline MBI $K_{\text {app }}(\mu \mathrm{M})$ & 74 & Experimental data \\
\hline MBI K $K_{\text {inact }}(1 / h)$ & 0.019 & Experimental data \\
\hline \multicolumn{3}{|l|}{ CYP3A4 enzyme induction } \\
\hline Ind $_{\max }$ & 20 & Experimental data \\
\hline Ind $\mathrm{EC}_{50}(\mu \mathrm{M})$ & 2 & Estimated based on clinical data \\
\hline P-gp inhibition $\mathrm{K}_{\mathrm{i}}(\mu \mathrm{M})$ & 0.5 & Estimated based on clinical data \\
\hline
\end{tabular}

$A D A M$ advanced dissolution, absorption, and metabolism, $C L / F$ apparent clearance, $C L_{\text {int }}$ intrinsic clearance, $C L_{\text {renal }}$ renal clearance, $C Y P$ cytochrome P450, $E C_{50}$ half maximal effective concentration, $f u$ fraction of unbound drug in plasma, $I n d_{\max }$ maximum induction, $J_{\max }$ maximal transport velocity, $K_{a p p}$ apparent binding constant, $K_{i}$ inhibitory constant, $K_{\text {inact }}$ inactivation rate constant, $K_{m}$ Michaelis-Menten constant, $\log P$ partition coefficient, $M B I$ mechanism-based inactivation, OATP organic anion transporting polypeptide, $P B P K$ physiologically based pharmacokinetic, $P$-g $p$ P-glycoprotein, $P K$ pharmacokinetic, $p K a$ acid dissociation constant, $V_{s s}$ volume of distribution at steady state 
with a single dose of rifampin (inhibitor of the OATP1B1 transporter) [4]. The elagolix-rifampin DDI data were used to optimize the in vitro maximal transport velocity $\left(J_{\max }\right)$ parameter for OATP1B1. Sensitivity analysis (electronic supplementary Fig. 1) was conducted to refine the final $J_{\max }$ estimate to improve the predictive performance of the PBPK model for the elagolix DDI with rifampin. The contribution of CYP3A4 to the overall elimination of elagolix was optimized using data from a multiple-dose rifampin DDI study [3]. The CYP3A4 intrinsic clearance $\left(\mathrm{CL}_{\text {int }}\right)$ estimate that helped improve the predictive performance of the PBPK model for the elagolix-rifampin DDI (following multiple doses) study was identified as the final parameter estimate in the model. At this step, verification of the model was conducted according to Shebley et al., defined as establishing consistency between the input parameters and underlying mechanisms and assumptions, and the ability of the model to successfully simulate independent sets of observed data [11]. The CYP3A4 contribution was verified using clinical data from the ketoconazole-elagolix DDI study by conducting sensitivity analysis (electronic supplementary Fig. 2). The overall hepatic metabolism of elagolix in the model was verified by comparing the model-predicted impact of $\mathrm{HI}$ on elagolix exposures with clinical data in the HI population.

As a perpetrator, elagolix inhibits P-gp and induces CYP3A4. The perpetrator (or interaction) model of elagolix was optimized (Table 1) using clinical data from DDI studies with digoxin (P-gp substrate) and midazolam (CYP3A substrate) [3]. To improve the predictive performance of the PBPK model, the in vitro induction parameter (half maximal effective concentration $\left[\mathrm{EC}_{50}\right]$ ) for elagolix was optimized using the clinical DDI study of elagolix with midazolam. Similarly, the digoxin-elagolix DDI study was used to optimize the in vitro $\mathrm{P}-\mathrm{gp}$ inhibition (inhibitory constant $\left[\mathrm{K}_{\mathrm{i}}\right]$ ) parameter.

\subsection{Simulation Design}

All simulations were performed using the default SimCYP ${ }^{\circledR}$ virtual Sim-Healthy Population representative (electronic supplementary Table 1). For all simulations, the physiological parameters for a healthy population were the same as reported by $\operatorname{SimCYP}^{\circledR}$ (version 15.0.86.0). For the HI study, the HI population model files (Child-Pugh A, B, and C) in SimCYP ${ }^{\circledR}$ were used. All population files were used without any modifications.

The expression of P-gp was the only physiological parameter that was altered for the elagolix-rifampin DDI simulations. Rifampin has been reported to induce P-gp expression four folds in in vitro and clinical studies [14, 15]. The four folds induction is known to predict digoxin DDIs [16], and hence P-gp expression in the liver and the intestine was

\section{Model Development}

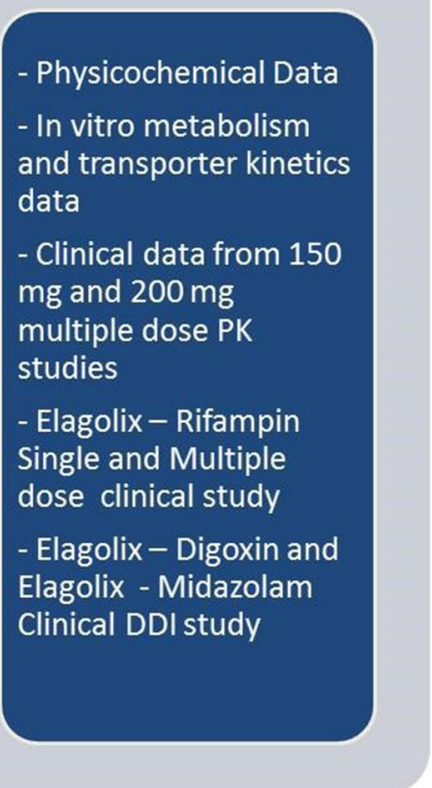

Model Verification

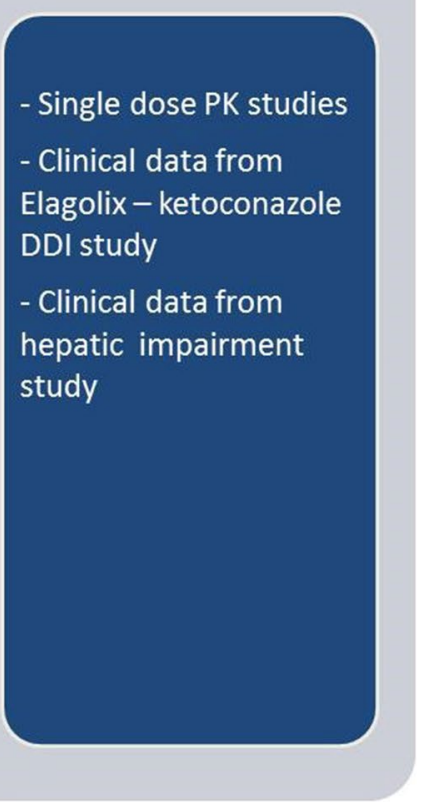

Model Application

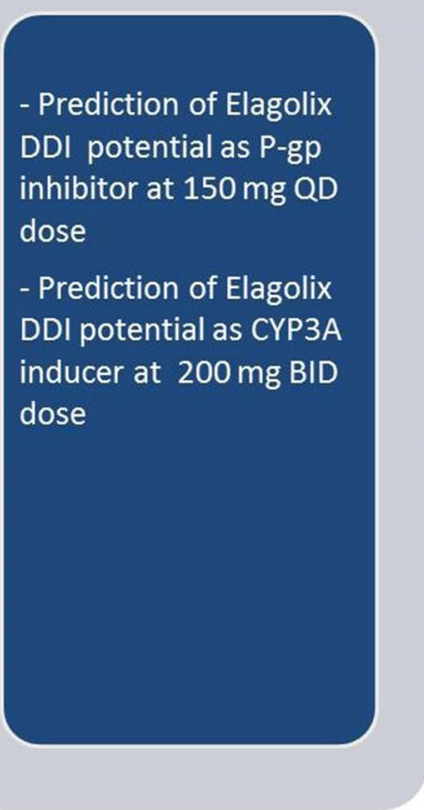

Fig. 1 Overview of the physiologically based pharmacokinetic model development, verification, and application strategy 
increased four folds on day 10 of the rifampin-elagolix DDI study using the methodology reported by Neuhoff et al. [16]. Following incorporation of the four folds increase in P-gp expression, the model-predicted digoxin $C_{\max }$ ratio was compared with the observed clinical data (electronic supplementary Fig. 3).

The study design used for the simulations was similar to the reported clinical study designs with regard to dose, route of administration, study duration, and fasting conditions (electronic supplementary Table 2).

\subsection{PBPK Model Performance Evaluation}

\subsubsection{Prespecified Acceptance Criteria for Pharmacokinetic Parameters (Maximum Concentration $\left[C_{\text {max }}\right]$, Area Under the Concentration-Time Curve [AUC], and Half-Life)}

The ability of the PBPK model to predict the observed clinical data of phase I PK studies was evaluated and quantified based on the percentage prediction error (\%PE). Predefined rules for acceptable \%PE were set, and the model was optimized during model development until the \% $\mathrm{PE}$ criteria were met. The \%PE was calculated using the formula described below (Eq. 1):

$\% \mathrm{PE}=\frac{\text { (predicted mean }- \text { observed mean) }}{\text { observed mean }} \times 100$

Based on the publication by Abduljalil et al. [17], the elagolix model predicted PK parameters were deemed to be within the acceptable limit of prediction if the \%PE was $\leq 50 \%$.

\subsubsection{Prespecified Acceptance Criteria for Drug-Drug Interaction (DDI) Studies and Hepatic Impairment Studies ( $C_{\max }$ Ratio and AUC Ratio)}

The model acceptance criterion for DDI studies was based on the publication by Guest et al. [18] A variable prediction margin dependent on the observed magnitude of DDIs was used for assessing the success of model prediction. The model was deemed to be acceptable if the predicted $C_{\max }$ and AUC ratios were within the defined range of success criteria. The limits for $C_{\max }$ and AUC ratios were calculated using the formula below (Eq. 2).

Limit $=\frac{1+2\left(R_{\mathrm{obs}}-1\right)}{R_{\mathrm{obs}}}$

where $R_{\text {obs }}=$ observed DDI ratio and is $>1$. For induction: $R_{\mathrm{obs}}=1 /$ (observed DDI ratio), Upper limit for acceptance $=R_{\mathrm{obs}} \times$ Limit, Lower limit of acceptance $=R_{\mathrm{obs}} /$ Limit

\section{Results}

\subsection{Development and Verification of the Elagolix PBPK Model Using a Combined Bottom-Up and Top-Down Approach}

The PBPK model of elagolix was developed using in vitro and clinical data. The PBPK model was able to adequately describe the PK profile (Fig. 2) and parameters (Table 2) of elagolix following doses of $150 \mathrm{mg}$ QD and $200 \mathrm{mg}$ BID $(\% \mathrm{PE}<12 \%)$. The contribution of OATP1B1 in the uptake of elagolix was optimized using the single-dose rifampin DDI study with elagolix [19]. Following model optimization, the concentration-time profiles with and without rifampin were well described by the model both visually (Fig. 2a) and quantitatively (Fig. 2b). The simulated elagolix $C_{\max }$ and AUC ratios were 3.2 and 4.0, respectively, on day 1 of rifampin coadministration (Table 3 ). While the predicted $C_{\max }$ and AUC ratios were within the predefined acceptance criterion, $28 \%$ underprediction by the model was observed. The CYP3A4 and P-gp contributions were optimized using clinical data from the administration of elagolix $150 \mathrm{mg}$ with multiple doses of rifampin $600 \mathrm{mg}$ QD [19]. The modelsimulated $C_{\max }$ and AUC ratios on day 10 were 2.28 and 2.29, respectively, and matched the observed $C_{\max }$ and AUC ratios of 2.0 and 1.65 , respectively (Table 3, Fig. 2).

The contribution of the CYP3A4 and P-gp pathway in the overall disposition of elagolix was verified independently using the DDI study with ketoconazole [19]. The modelsimulated $C_{\max }$ and AUC ratios of elagolix were 2.01 and 2.13, respectively, and the observed $C_{\max }$ and AUC ratios were 1.77 and 2.2, respectively (Table 3, Fig. 3a). The observed and predicted ratios were within the limits of the model acceptance criterion (Table 3, Fig. 4).

The assignment of hepatic metabolism to overall clearance of elagolix in the PBPK model was verified using clinical data from the HI studies. Based on the PBPK model simulations, the predicted ratio of elagolix AUC in Child-Pugh A, B, and $\mathrm{C}$ patients relative to healthy volunteers was $1.6,4.5$, and 7.3, respectively, while the clinically observed AUC ratios were $0.8,2.7$, and 6.7, respectively (Table 3). For $C_{\max }$, the modelpredicted ratios in Child-Pugh A, B, and C patients relative to healthy volunteers were $1.5,3.7$, and 4.8 , respectively, while the clinically observed $C_{\max }$ ratios were $0.8,2.6$, and 6.2 , respectively (Table 3, Fig. 3b). The final PBPK model of elagolix as a victim reasonably captured the interplay between CYP3A4, P-gp, and OATP1B1 based on comparisons of the model simulation results with clinically observed PK, DDI ratios, and impact of HI. At this stage, the elagolix PBPK victim model was qualified for evaluation of the impact of extrinsic factors on modulation of multiple ADME mechanisms involved in the disposition of elagolix (Fig. 4). 
Table 2 Elagolix PBPK model development: predicted vs. observed pharmacokinetic parameters of elagolix following multiple doses in healthy subjects

\begin{tabular}{|c|c|c|c|c|c|c|}
\hline \multirow{2}{*}{$\begin{array}{l}\text { Pharmacokinetic } \\
\text { parameter (units) }\end{array}$} & \multicolumn{3}{|c|}{$150 \mathrm{mg}$ QD $(N=6)$} & \multicolumn{3}{|c|}{$200 \mathrm{mg} \operatorname{BID}(N=7)$} \\
\hline & Predicted & Observed & $\% \mathrm{PE}$ & Predicted & Observed & $\% \mathrm{PE}$ \\
\hline \multicolumn{7}{|l|}{ Day 1} \\
\hline$C_{\max }(\mathrm{ng} / \mathrm{mL})$ & 524 & 507 & 3.4 & 779 & 712 & 9.4 \\
\hline $\mathrm{T}_{\max }(\mathrm{h})$ & 0.95 & 1.3 & 26.9 & 0.95 & 1.0 & 5.0 \\
\hline $\mathrm{AUC}_{\tau}(\mathrm{ng} \bullet \mathrm{h} / \mathrm{mL})^{\mathrm{a}}$ & 1321 & 1331 & 0.8 & 1756 & 1813 & 3.1 \\
\hline \multicolumn{7}{|l|}{ Day 21} \\
\hline$C_{\max }(\mathrm{ng} / \mathrm{mL})$ & 524 & 574 & 3.4 & 680 & 774 & 12.1 \\
\hline $\mathrm{AUC}_{\tau}(\mathrm{ng} \bullet \mathrm{h} / \mathrm{mL})^{\mathrm{a}}$ & 1213 & 1292 & 6.1 & 1518 & 1725 & 12 \\
\hline $\mathrm{CL} / \mathrm{F}(\mathrm{L} / \mathrm{h})$ & 124 & 123 & 1 & 132 & 144 & 8 \\
\hline$t_{1 / 2}(h)$ & 4.57 & 6.42 & 28.8 & 4.54 & 4.29 & 5.8 \\
\hline
\end{tabular}

$A U C_{\tau}$ area under the curve over the dosing interval, bid twice daily, $C_{\max }$ maximum concentration, $P B P K$ physiologically based pharmacokinetic, $\% P E$ percentage prediction error, $q d$ once daily, $T_{\max }$ time to maximum concentration, $t_{1 / 2}$ terminal elimination half-life

\subsection{PBPK Model of Elagolix as a Cytochrome P450 (CYP) 3A Inducer and P-Glygoprotein (P-gp) Inhibitor}

The in vitro CYP3A induction data of elagolix, when used in the PBPK model, was not able to quantitatively predict the observed $C_{\max }$ and AUC ratios of midazolam (results not shown). To improve the predictive performance of the PBPK model, the in vitro induction parameter $\left(\mathrm{EC}_{50}\right)$ for elagolix was optimized by approximately threefold. Following optimization, the model-predicted AUC ratio of 0.69 closely matched the observed AUC ratio of 0.65 (Table 4, Fig. 4). Similarly, for the digoxin-elagolix DDI study, the in vitro P-gp inhibition $\left(K_{\mathrm{i}}\right)$ parameter of elagolix was optimized. Digoxin day 1 and day 10 simulated $C_{\max }$ ratios were 1.74 and 1.73, respectively, and the AUC ratios were 1.22 and 1.19 , respectively. These model results were in good agreement with the observed day 1 and day $10 C_{\max }$ ratios (1.73 and 1.71) and the day 1 and day 10 AUC ratios (1.32 and 1.26) (Table 4, Fig. 4) [19]. Based on the simulation results, the PBPK perpetrator model was considered to be qualified for assessing the DDI potential of elagolix as a perpetrator for CYP3A and P-gp substrates.

\subsection{PBPK Model Application: Prediction of the DDI Potential of Elagolix as a CYP3A4 and P-gp Perpetrator}

The final and verified PBPK model of elagolix was used to predict DDI with midazolam at the higher approved dose of $200 \mathrm{mg}$ BID. The AUC of midazolam was predicted to decrease by $56 \%$, while the $C_{\max }$ was predicted to decrease by $49 \%$ (Table 4 ), following coadministration with elagolix $200 \mathrm{mg}$ BID. The CYP3A4 induction simulation results enabled characterization of elagolix as a moderate inducer at the higher 200 mg BID dose, which was not evaluated in DDI trials, and informed the labeling language for coadministration with CYP3A substrates [19].

Similarly, the final model was used to predict changes in the $C_{\max }$ and AUC of digoxin, following multiple $150 \mathrm{mg}$ QD doses of elagolix. At $150 \mathrm{mg}$ QD, the $C_{\max }$ and AUC of digoxin were predicted to increase by $68 \%$ and $19 \%$, respectively, on day 1, while, on day 10 , the $C_{\max }$ and AUC of digoxin were predicted to increase by $73 \%$ and $19 \%$, respectively (Table 4). These results indicated that elagolix inhibition of P-gp may not be dose- or time-dependent within the evaluated dose range.

\section{Discussion}

The PBPK model of elagolix was developed to mechanistically account for all of the known disposition mechanisms of elagolix (i.e. quantify the interplay between metabolism by CYP3A4, hepatic uptake by OATP1B1, and intestinal efflux by P-gp) and to support DDI dosing recommendations for the coadministration of elagolix with other drugs such as midazolam (CYP3A substrate) and digoxin (P-gp substrate). Elagolix has two approved dosages (150 mg QD and $200 \mathrm{mg}$ BID) for the treatment of endometriosis, and DDI studies were conducted at either $150 \mathrm{mg}$ QD or $200 \mathrm{mg}$ BID for midazolam or digoxin, respectively.

The mechanisms involved in the disposition of elagolix were incorporated in the PBPK model using clinical DDI studies of elagolix with rifampin and ketoconazole. The interplay between the uptake transporter OATP1B1, efflux transporter P-gp, and drug-metabolizing enzyme CYP3A4 was captured using data from the elagolix-rifampin DDI study. Following a single dose, rifampin inhibits OATP1B1 and P-gp; however, following multiple doses, rifampin also induces CYP3A and P-gp [4]. The relative contribution of OATP1B1 and P-gp in the transport of elagolix was 
Fig. 2 PBPK model development. a Predicted vs. observed mean concentration-time profiles of elagolix $(150 \mathrm{mg}$ QD) with and without coadministration of rifampin $(600 \mathrm{mg}$ QD). b Predicted vs. observed concentrations of elagolix from multiple-dose PK and DDI studies. $D D I$ drug-drug interaction, $P B P K$ physiologically based pharmacokinetic, $P K$ pharmacokinetic, $Q D$ once daily
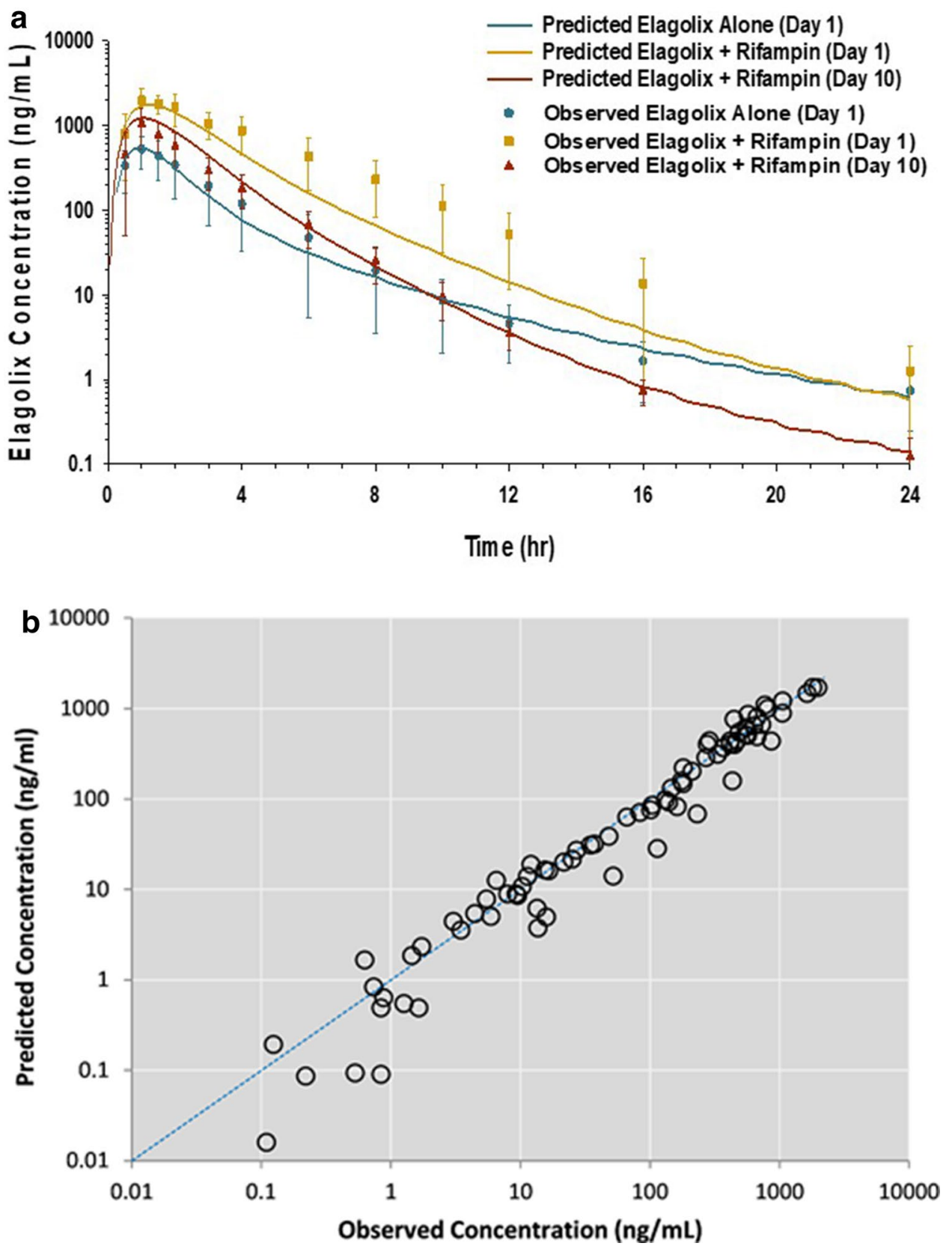

optimized using clinical data following coadministration of a single dose of rifampin with elagolix. The AUC of elagolix was sensitive to the OATP1B1 maximal rate of transport $\left(J_{\max }\right)$, and the optimized value of $J_{\max }$ was selected to match the observed elagolix AUC and $C_{\max }$ (electronic supplementary Fig. 1). The contribution of OATP1B1 and $\mathrm{P}$-gp was fixed, and clinical data following multiple doses of rifampin coadministered with a single dose of elagolix were used to optimize the CYP3A4 and P-gp contributions. This approach of leveraging clinical DDI data following single and multiple doses of rifampin was recently used by Asaumi et al. to predict the complex DDIs of rifampin with glibenclamide [20]. Given that the clinical DDI study of rifampin and elagolix was used to identify multiple parameters, the contribution of CYP3A4 and P-gp to the overall disposition of elagolix was independently verified using clinical data from the elagolix-ketoconazole DDI study to mitigate concerns regarding parameter identifiability (electronic supplementary Fig. 2). However, the OATP1B1 contribution could not be verified using an independent dataset since additional DDI studies of elagolix with an OATP1B1 inhibitor are not available. Nonetheless, the model-predicted exposures in the OATP1B1 phenotypes (extensive transporter, intermediate transporter, and poor transporter) matched the clinically observed exposures in subjects with matching OATP1B1 genotypes (data not shown), providing confidence 
Table 3 Elagolix PBPK model development and verification: predicted vs. observed $\boldsymbol{C}_{\text {max }}$ and AUC ratios following coadministration of elagolix with prototypical perpetrators and in patients with hepatic impairment

\begin{tabular}{|c|c|c|c|c|c|c|c|}
\hline & \multirow[t]{2}{*}{ Elagolix dose, mg } & \multicolumn{3}{|c|}{ Mean $C_{\max }$ ratio } & \multicolumn{3}{|c|}{ Mean AUC ratio } \\
\hline & & Predicted & Observed & $\% \mathrm{PE}$ & Predicted & Observed & $\% \mathrm{PE}$ \\
\hline $\begin{array}{l}\text { Coadministered } \\
\text { drug with } \\
\text { elagolix }\end{array}$ & DDI studies & & & & & & \\
\hline Rifampin day 1 & $150^{\mathrm{a}}$ & 3.20 & 4.37 & 27 & 4.00 & $5.58^{\mathrm{b}}$ & 28 \\
\hline Rifampin day 10 & $150^{\mathrm{a}}$ & 2.28 & 2.00 & 14 & 2.29 & $1.65^{\mathrm{b}}$ & 39 \\
\hline Ketoconazole & $150^{\mathrm{a}}$ & 2.01 & 1.77 & 14 & 2.13 & $2.20^{\mathrm{c}}$ & 3.2 \\
\hline Patient population & Hepatic impairment studies & & & & & & \\
\hline Child-Pugh A & $150^{\mathrm{a}}$ & 1.5 & 0.8 & 88 & 1.6 & $0.8^{\mathrm{c}}$ & 100 \\
\hline Child-Pugh B & $150^{\mathrm{a}}$ & 3.7 & 2.6 & 42 & 4.5 & $2.7^{\mathrm{c}}$ & 67 \\
\hline Child-Pugh C & $150^{\mathrm{a}}$ & 4.8 & 6.2 & -23 & 7.3 & $6.7^{\mathrm{c}}$ & 9 \\
\hline
\end{tabular}

$A U C$ area under the curve, $A U C_{24}$ AUC from time zero to $24 \mathrm{~h}, A U C_{\infty}$ AUC from time zero to infinity, $C_{\max }$ maximum concentration, $D D I$ drug-drug interaction, $P B P K$ physiologically based pharmacokinetic, $\% P E$ percentage prediction error (acceptance criteria)

${ }^{\text {a }}$ Single-dose elagolix

${ }^{\mathrm{b}} \mathrm{AUC}_{24}$

${ }^{\mathrm{c}} \mathrm{AUC}_{\infty}$

in the assignment of the relative contribution of OATP1B1 in the active hepatic uptake of elagolix.

The ability of the model to capture the overall hepatic elimination of elagolix was further verified using clinical data of elagolix from the HI study. The HI population in the PBPK model in SimCYP ${ }^{\circledR}$ version 15 accounts for most of the changes known to be associated with HI. The model reasonably predicted changes in the exposures of elagolix for Child-Pugh B and C patients, but overpredicted the exposures in Child-Pugh A patients. The differences in model predictions to the observed changes may be attributed to a combination of factors, such as a lack of information regarding the impact of $\mathrm{HI}$ on transporter expression, potential changes in the minor elimination pathways in the HI population that were not accounted for in the model, differences in the demographics of the study population, or the small sample size in the actual clinical study. The PBPK model predictions in the HI population are encouraging given that there is a growing interest in utilizing PBPK models to predict the impact of organ impairment on the PK of drugs. Morcos et al. used a PBPK model-based approach to provide recommendations regarding the study design of alectinib in patients with HI [21], while Pilla Reddy et al. used the PBPK model of olaparib to predict the exposures in patients with HI following multiple doses [22]. For obeticholic acid, a PBPK model was used to support regulatory recommendations regarding dose adjustment in $\mathrm{HI}$ patients. Taken together, the predictive ability of the elagolix PBPK model using external data from clinical pharmacology studies, including an HI study, indicates that the PBPK model performance of elagolix is robust for accurately predicting elagolix exposures at $150 \mathrm{mg}$ QD and $200 \mathrm{mg}$ BID.
While using the PBPK model to guide potential DDI risk assessment, a limitation of the model was the use of a single virtual population representative rather than a virtual population of subjects with varying intrinsic covariates. The population representative approach was selected to enable exploration of the implications of transporter-metabolism interplay on the PK of elagolix and the impact on coadministered CYP3A or P-gp substrates. In addition, the relative contribution of other minor metabolic pathways was incorporated in the PBPK model but were not verified in DDI studies with prototypical inhibitors. Future iterations of the model will investigate the influence of variability on elagolix PBPK simulations; however, this was beyond the scope of this application.

The key regulatory application of the PBPK model was to predict the DDI potential of elagolix at alternative doses that were not evaluated in the clinic (i.e. elagolix $200 \mathrm{mg}$ BID with midazolam, or elagolix $150 \mathrm{mg}$ QD with digoxin). The PBPK model was calibrated using the clinical data from DDI studies by optimizing the $\mathrm{EC}_{50}$ for CYP3A4 induction, and the $\mathrm{K}_{\mathrm{i}}$ for P-gp inhibition. While optimized parameters were able to predict the clinically observed DDI potential, there is uncertainty associated with these parameter estimates given that data from DDI studies with one dose level of elagolix (i.e. $150 \mathrm{mg}$ QD or $200 \mathrm{mg}$ BID) were used for estimation. PBPK modeling results indicated that elagolix decreases midazolam exposures by approximately $50 \%$ following administration of elagolix $200 \mathrm{mg}$ BID. These CYP3A4 induction simulation results enabled characterization of elagolix as a moderate inducer at the higher $200 \mathrm{mg}$ BID dose, which was not evaluated in DDI trials. 
Fig. 3 a PBPK model verification (elagolix as victim): predicted vs. observed mean concentration-time profiles of elagolix with and without coadministration of ketoconazole. b PBPK model verification: predicted vs. observed concentrations of elagolix from single-dose, DDI, and hepatic impairment studies. $D D I$ drug-drug interaction, $P B P K$ physiologically based pharmacokinetic
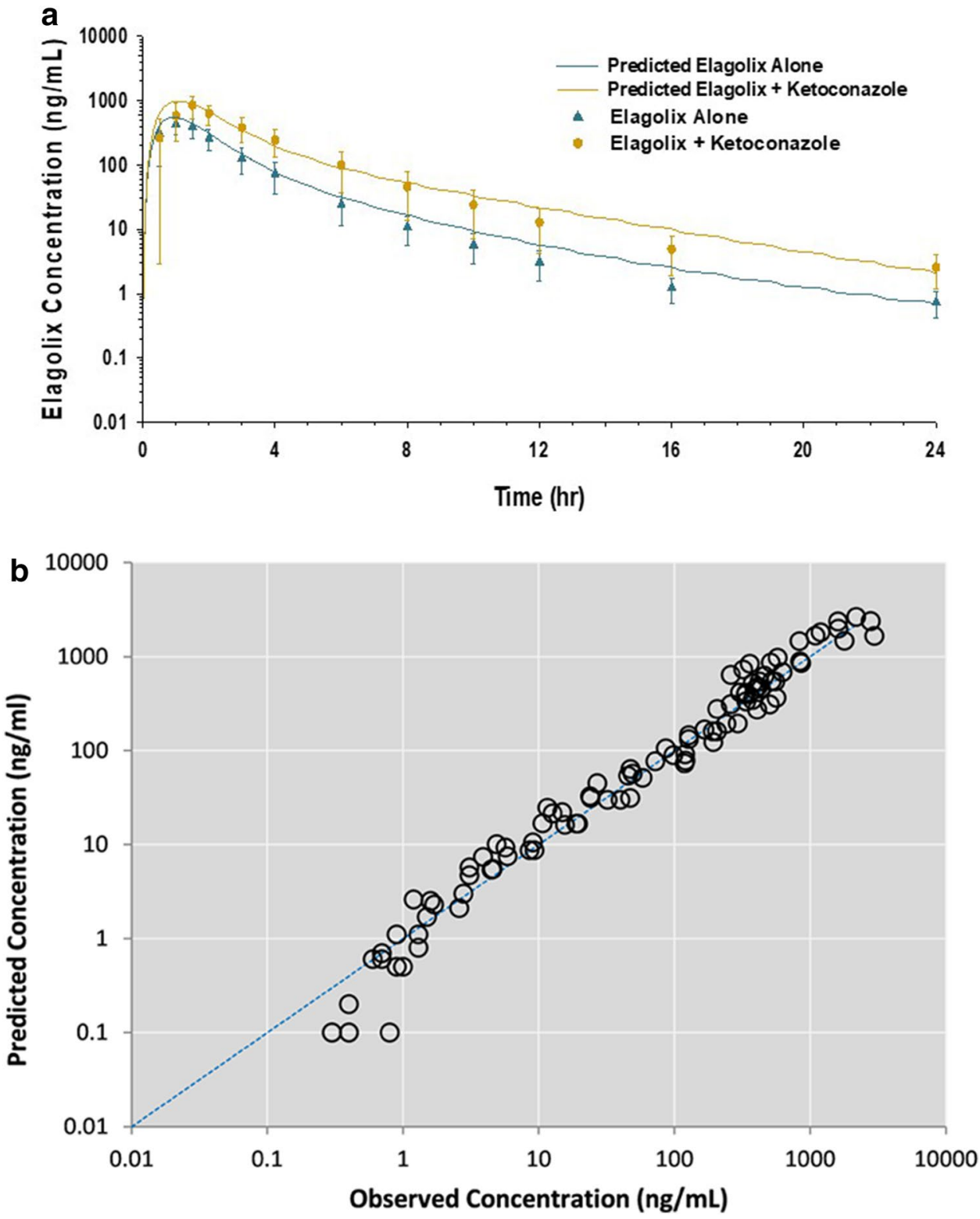

The model was also used to characterize the dose-dependent effects of elagolix on P-gp after single and multiple doses. The predicted magnitude of DDIs at $150 \mathrm{mg}$ QD is similar to the magnitude of DDIs clinically observed at 200 mg BID, suggesting that elagolix inhibition of P-gp may not be dose-dependent at this dose range of elagolix. This is anticipated given that the model-estimated $K_{\mathrm{i}}$ for P-gp was $0.5 \mu \mathrm{M}$, approximately 500-fold below the predicted intestinal elagolix concentration in the gastrointestinal lumen following administration of 150 or $200 \mathrm{mg}$ of elagolix. A similar extent of P-gp inhibition was predicted on days 1 and 10 following elagolix administration, consistent with the short half-life and lack of accumulation of elagolix. Overall, the results from the PBPK model were used to inform the labeling language of elagolix for coadministration with CYP3A and P-gp substrates.

\section{Conclusions}

A PBPK model of elagolix that captures the absorption using a mechanistic model, the interplay between metabolism by CYP3A4 in the liver and gut, OATP1B1-mediated uptake in the liver, and intestinal and hepatic efflux via the P-gp transporter, along with the induction and inhibition of CYP3A4 and P-gp by elagolix, was developed and verified using in vitro and clinical PK/DDI data. The PBPK model was utilized to inform the DDI potential of 


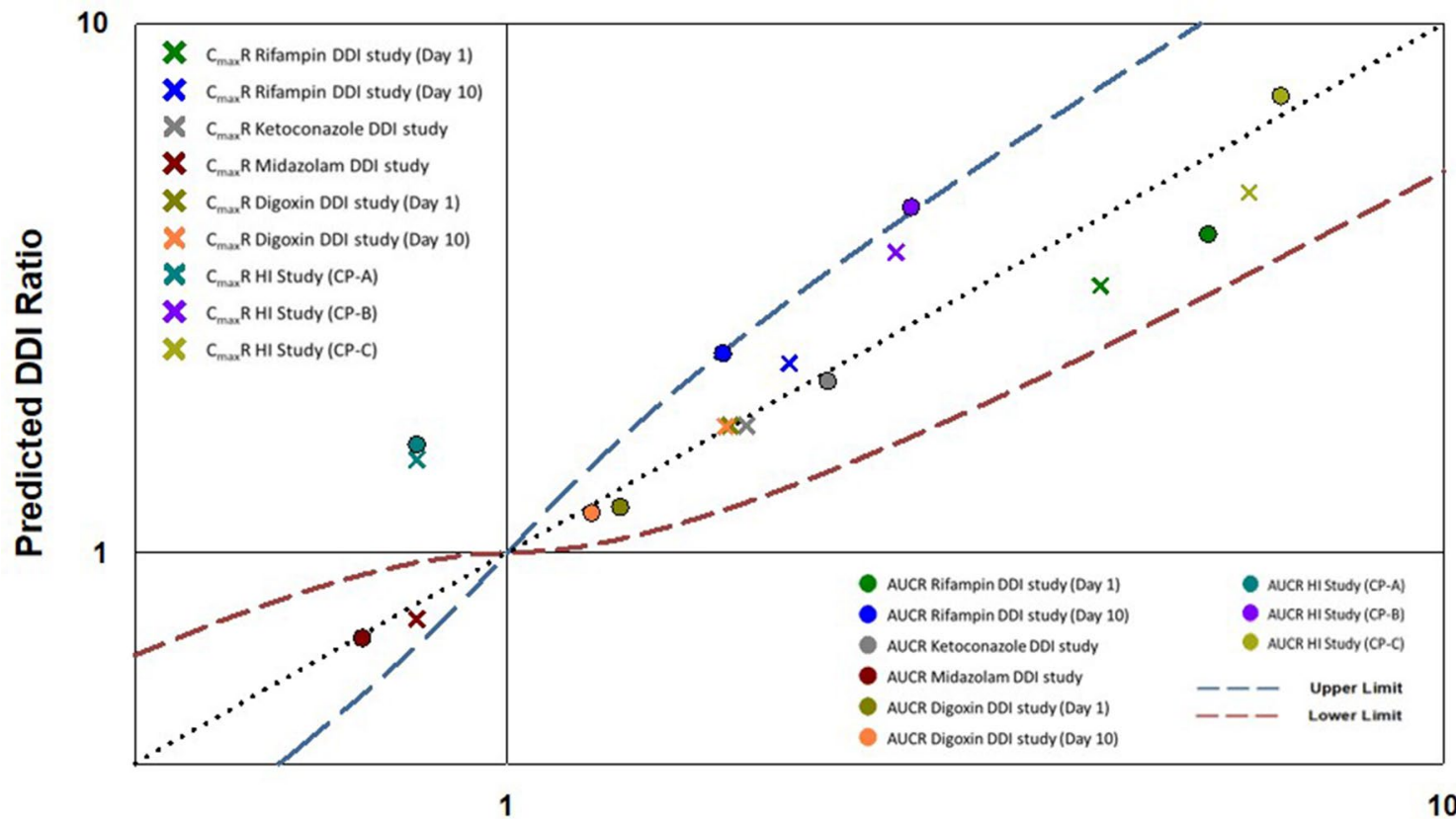

Observed DDI Ratio

Fig. 4 PBPK model performance: observed and predicted $C_{\max }$ and AUC ratio for elagolix DDI studies fall within the predefined limits of model acceptance criteria. $A U C$ area under the concentration-time curve, $C_{\max }$ maximum concentration, $D D I$ drug-drug interaction, $P B P K$ physiologically based pharmacokinetic

Table 4 PBPK model predictions: elagolix as a perpetrator

\begin{tabular}{|c|c|c|c|c|c|c|c|c|}
\hline \multirow[t]{2}{*}{ Victim drug } & \multirow[t]{2}{*}{ Model stage } & \multirow[t]{2}{*}{ Elagolix dose } & \multicolumn{3}{|c|}{ Mean $C_{\max }$ ratio } & \multicolumn{3}{|c|}{ Mean $\mathrm{AUC}_{\infty}$ ratio } \\
\hline & & & Predicted & Observed & $\% \mathrm{PE}(\%)$ & Predicted & Observed & $\% \mathrm{PE}(\%)$ \\
\hline \multirow[t]{2}{*}{ Midazolam } & Development & $150 \mathrm{mg}$ QD & 0.75 & 0.81 & $8(77)$ & 0.69 & 0.65 & $6(46)$ \\
\hline & Application & $200 \mathrm{mg}$ BID & 0.51 & Not applicable & & 0.44 & Not applicable & \\
\hline Digoxin, day 1 & Development & $200 \mathrm{mg}$ BID & 1.74 & 1.73 & $0.6(42)$ & 1.22 & 1.32 & $8(24)$ \\
\hline Digoxin, day 10 & & $200 \mathrm{mg}$ BID & 1.73 & 1.71 & $1(42)$ & 1.19 & 1.26 & $6(21)$ \\
\hline Digoxin, day 1 & Application & $150 \mathrm{mg}$ QD & 1.68 & Not applicable & & 1.19 & Not applicable & \\
\hline Digoxin, day 10 & & $150 \mathrm{mg}$ QD & 1.73 & & & 1.19 & & \\
\hline
\end{tabular}

$A U C_{\infty}$ area under the curve from time zero to infinity, BID twice daily, $C_{\max }$ maximum concentration, $P B P K$ physiologically based pharmacokinetic, $\% P E$ percentage prediction error (acceptance criteria), $Q D$ once daily

PBPK simulation trial design for midazolam: elagolix administered for 16 days, midazolam administered on day 14 as a single dose PBPK simulation trial design for digoxin: elagolix administered for 13 days, digoxin administered on days 1 and 10 as a single dose

elagolix as a perpetrator for CYP3A and P-gp substrates for doses at which clinical data were not available. Based on model predictions, elagolix is a weak to moderate inducer of CYP3A. Coadministration with elagolix may decrease plasma concentrations of drugs that are sensitive substrates of CYP3A. Elagolix is an inhibitor of the efflux transporter $\mathrm{P}$-gp, and clinical monitoring for digoxin is recommended when coadministered with elagolix. This clinically verified PBPK model of elagolix may be used for future applications and regulatory submissions.
Acknowledgments All authors contributed to the study design, research, and interpretation of data, and to the writing, reviewing, and approval of the publication. Medical writing support was provided by Amy Rohrlack and Wesley Wayman, employees of AbbVie.

\section{Compliance with Ethical Standards}

Funding This study was funded by AbbVie.

Conflict of interest Manoj S. Chiney, Juki Ng, John P. Gibbs, and Mohamad Shebley are employees and shareholders of AbbVie. 
Data Sharing Statement AbbVie is committed to responsible data sharing regarding the clinical trials we sponsor. This includes access to anonymized, individual and trial-level data (analysis data sets), as well as other information (e.g. protocols and clinical study reports), as long as the trials are not part of an ongoing or planned regulatory submission. This includes requests for clinical trial data for unlicensed products and indications. These clinical trial data can be requested by any qualified researchers who engage in rigorous, independent scientific research, and will be provided following review and approval of a research proposal and Statistical Analysis Plan (SAP) and execution of a Data Sharing Agreement (DSA). Data requests can be submitted at any time and the data will be accessible for 12 months, with possible extensions considered. For more information on the process, or to submit a request, visit the following link: https://www.abbvie.com/ our-science/clinical-trials/clinical-trials-data-and-information-sharing/ data-and-information-sharing-with-qualified-researchers.html.

Open Access This article is distributed under the terms of the Creative Commons Attribution-NonCommercial 4.0 International License (http://creativecommons.org/licenses/by-nc/4.0/), which permits any noncommercial use, distribution, and reproduction in any medium, provided you give appropriate credit to the original author(s) and the source, provide a link to the Creative Commons license, and indicate if changes were made.

\section{References}

1. Ng J, Chwalisz K, Carter DC, Klein CE. Dose-dependent suppression of gonadotropins and ovarian hormones by elagolix in healthy premenopausal women. J Clin Endocrinol Metab. 2017;102:1683-91.

2. Taylor HS, Giudice LC, Lessey BA, Abrao MS, Kotarski J, Archer $\mathrm{DF}$, et al. Treatment of endometriosis-associated pain with elagolix, an oral GnRH antagonist. N Engl J Med. 2017;377:28-40.

3. Shebley M, Polepally AR, Nader A, Ng JW, Winzenborg I, Klein $\mathrm{CE}$, et al. Clinical pharmacology of elagolix: an oral gonadotropin-releasing hormone receptor antagonist for endometriosis. Clin Pharmacokin. 2019. https://doi.org/10.1007/s40262-019-00840-7.

4. Niemi M, Backman JT, Fromm MF, Neuvonen PJ, Kivisto KT. Pharmacokinetic interactions with rifampicin: clinical relevance. Clin Pharmacokinet. 2003;42:819-50.

5. Ng J, Duan WR, Marbury T, Schmidt JM, Klein CE. Elagolix pharmacokinetic profiles in women with renal or hepatic impairment. Clin Pharmacol Drug Dev. 2018. https://doi.org/10.1002/ cpdd.640.

6. Polepally AR, Dufek MB, Dharia SP, Kamradt K, Lin J, Peloso PM, et al. Drug-drug interaction of midazolam with elagolix, a novel oral GnRH antagonist: analyses of data from two studies. Clin Pharmacol Drug Dev. 2017;6:48.

7. Ng J, Salem A, Carter D, Klein CE. Effects of the coadministration of multiple doses of elagolix on the pharmacokinetics and safety of digoxin in healthy women. Clin Pharmacol Drug Dev. 2017;6:54-5.

8. Zhao P, Zhang L, Grillo JA, Liu Q, Bullock JM, Moon YJ, et al. Applications of physiologically based pharmacokinetic (PBPK) modeling and simulation during regulatory review. Clin Pharmacol Ther. 2011;89:259-67.
9. Huang SM. PBPK as a tool in regulatory review. Biopharm Drug Dispos. 2012;33:51-2.

10. Arya V, Zhao P, Reynolds KS, Mishra P, Younis IR. Utilizing PBPK modeling to evaluate the potential of a significant drugdrug interaction between clopidogrel and dasabuvir: a scientific perspective. Clin Pharmacol Ther. 2017;102:578-80.

11. Shebley M, Sandhu P, Emami Riedmaier A, Jamei M, Narayanan R, Patel A, et al. Physiologically based pharmacokinetic model qualification and reporting procedures for regulatory submissions: a consortium perspective. Clin Pharmacol Ther. 2018;104(1):88-110.

12. Freise KJ, Shebley M, Salem AH. Quantitative prediction of the effect of CYP3A inhibitors and inducers on venetoclax pharmacokinetics using a physiologically based pharmacokinetic model. J Clin Pharmacol. 2017;57:796-804.

13. Rodgers T, Rowland M. Mechanistic approaches to volume of distribution predictions: understanding the processes. Pharm Res. 2007;24:918-33.

14. Greiner B, Eichelbaum M, Fritz P, Kreichgauer HP, von Richter O, Zundler J, et al. The role of intestinal P-glycoprotein in the interaction of digoxin and rifampin. J Clin Invest. 1999;104:147-53.

15. Westphal K, Weinbrenner A, Zschiesche M, Franke G, Knoke M, Oertel R, et al. Induction of P-glycoprotein by rifampin increases intestinal secretion of talinolol in human beings: a new type of drug/drug interaction. Clin Pharmacol Ther. 2000;68:345-55.

16. Neuhoff S, Yeo KR, Barter Z, Jamei M, Turner DB, RostamiHodjegan A. Application of permeability-limited physiologicallybased pharmacokinetic models: part II-prediction of P-glycoprotein mediated drug-drug interactions with digoxin. J Pharm Sci. 2013;102:3161-73.

17. Abduljalil K, Cain T, Humphries H, Rostami-Hodjegan A. Deciding on success criteria for predictability of pharmacokinetic parameters from in vitro studies: an analysis based on in vivo observations. Drug Metab Dispos. 2014;42:1478-84.

18. Guest EJ, Aarons L, Houston JB, Rostami-Hodjegan A, Galetin A. Critique of the two-fold measure of prediction success for ratios: application for the assessment of drug-drug interactions. Drug Metab Dispos. 2011;39:170-3.

19. AbbVie Inc. Orilissa (elagolix tablets) [US package insert]. North Chicago: AbbVie Inc., 2018.

20. Asaumi R, Toshimoto K, Tobe Y, Hashizume K, Nunoya K, Imawaka $\mathrm{H}$, et al. Comprehensive PBPK model of rifampicin for quantitative prediction of complex drug-drug interactions: CYP3A/2C9 induction and OATP inhibition effects. CPT Pharmacometr Syst Pharmacol. 2018;7:186-96.

21. Morcos PN, Cleary Y, Sturm-Pellanda C, Guerini E, Abt M, Donzelli M, et al. Effect of hepatic impairment on the pharmacokinetics of alectinib. J Clin Pharmacol. 2018;58:1618-28.

22. Pilla Reddy V, Bui K, Scarfe G, Zhou D, Learoyd M. Physiologically based pharmacokinetic modeling for olaparib dosing recommendations: bridging formulations, drug interactions, and patient populations. Clin Pharmacol Ther. 2019;105:229-41.

23. Jamei M, Turner D, Yang J, Neuhoff S, Polak S, Rostami-Hodjegan A. Population-based mechanistic prediction of oral drug absorption. AAPS J. 2009;11:225-37. 